\title{
Ultrasonography-Guided Percutaneous Nephrolithotomy for the Treatment of Kidney Stone
}

\author{
Hong-yan $\mathrm{LI}^{1}$, Hai LI ${ }^{2}$ and Yuan-yuan $\mathrm{XING}^{3,{ }^{*}}$ \\ 'Department of Urology, China-Japan Union Hospital of Jilin University, Changchun \\ 130033, Jilin, China \\ Tel: +86-13756069386 Email: Lihongyan006@163.com \\ ${ }^{2} \mathrm{Hai}$ Li:Department of Urology, China-Japan Union Hospital of Jilin University, \\ Changchun 130033, Jilin, China \\ ${ }^{3}$ Department of Dialysis centers, China-Japan Union Hospital of Jilin University, \\ Changchun 130033, Jilin, China \\ Tel: +86-13756069387; Fax: +86-431-89876971. Email: 624177683@qq.com \\ ${ }^{*}$ Corresponding author
}

Keywords: Complicating urolithiasis, Percutaneous nephrolithotomy, Minimally invasive, Effectiveness, Safety.

\begin{abstract}
We examined the surgical outcomes of minimally invasive percutaneous nephrolithotomy (MPCNL) in complicating urolithiasis.10 patients were hospitalized for MPNCL due to upper tract urolithiasis. Calyx puncture was performed in the prone position under ultrasonographic guidance. The renal access route was established using a set of $8 \mathrm{~F}-12 \mathrm{~F}$ dilators, and a transpyelic ballistic lithotriptor was used to fragment calculi. The entire operative time was approximately 40-60 min, and the mean time of establishing percutaneous access was $20 \mathrm{~min}$. The calculi were completely removed by single-session pneumatic lithotripsy. The 10 patients recovered from MPCNL uneventfully, and the follow-up radiological examinations identified no stone residual or recurrence.MPCNL is a minimally invasive modality that is effective and safe for the treatment of urolithiasis in patients.
\end{abstract}

\section{Introduction}

Upper tract urolithiasis (UTUL) or upper urinary calculosis is a common urological disorder that is rapidly increasing in the Chinese population. Surgical intervention is usually required in serious or complicated cases; however, the conventional open procedure imparts a heavy burden on the patient physiologically, psychologically and financially. In many cases minimally invasive surgery (MIS) has been successfully used to treat UTUL. Multiple methods exist, including extracorporeal shock wave lithotripsy (ESWL), antegrade/retrograde ureteroscopy (A/RUS), percutaneous nephrolithotomy (PCNL), and laparoscopy. [1] In patients with larger calculi (diameter $>2 \mathrm{~cm}$ ), neither ESWL nor A/RUS is adequate and associated with a greater risk of morbidity. [2] PCNL has become the standard treatment of care for complicated UTUL due to its high success rate and low complication rate. [3]Additionally, PCNL has been successful in treating patients with failed open surgery or ESWL, kidney transplantation, and urinary deformity or diversion. [4,7]

PCNL is contraindicated for patients with obesity, pelvic kidney, or spinal deformities, such as scoliosis, kyphosis and lordosis. [2] These pre-existing conditions 
will result in movement of anatomical landmarks and consequently increase the frequency of procedural complications in establishing percutaneous renal access. Furthermore, scoliotic patients may suffer from more serious urolithiasis, probably in association with the underlying congenital anomalies or secondary variations in the urinary tract. [8] Kara et al. [9] reported successful treatment of a small series of previously-treated scoliotic patients suffering from large renal stones in a single center by applying PCNL and auxiliary ureteroscopy. Their experience suggests that PCNL may be suitable for patients with scoliosis.

In 1992, Wu et al. [10]developed a modified PCNL, termed minimally invasive PCNL (MPCNL), that has been found valuable for treating urinary calculosis. This technique has been well recognized in urological practice due to its low morbidity rate and broad indications. In this study, we examined the feasibility, effectiveness, and safety of MPCNL for the treatment of refractory UTUL in patients with concomitant congenital scoliosis.

\section{Methods}

\section{Patients and Assessments}

10 patients were referred to our urology center due to radiologically diagnosed UTUL and aggravated symptoms between February 2015 and March 2016. The predominant complaint/symptom was intermittent flank pain in both patients.

Preoperative radiological assessments consisted of kidney-ureter-bladder (KUB) radiography, abdominal non-contrast-enhanced computed tomography (CT), and intravenous urography (IVU).

The ten patients gave informed consent prior to MPCNL. The same attending urologist performed the two procedures and was assisted by an assigned team of assistant surgeons, anesthesiologists, and surgical nurses. The patients were intubated and positioned in the lithotomy position under combined intravenous-inhalational general anesthesia. A 5F ureteral access catheter was induced into the diseased ureter with the assistance of ureteroscopy (uroVision GmbH, Bad Aibling, Germany), and the distal end of the ureteral catheter was secured to an $18 \mathrm{~F}$ Foley bladder catheter. The patients were turned to the prone position, with the abdomen cushioned and the waist elevated.

Demographic and clinical data for two of the ten patients are presented in Table 1. The Cobb angle, a measure used to describe the coronal plane deformity on antero-posterior plain radiographs in the classification of scoliosis, was determined to be $51^{\circ}$ in patient No. 1 and $58^{\circ}$ in patient No. 2, respectively. The stone burdens were $410 \mathrm{~mm}^{2}$ and $500 \mathrm{~mm}^{2}$, respectively. An 18-gauge access needle (uroVision $\mathrm{GmbH}$, Bad Aibling, Germany) was inserted percutaneously through the intersection of the 11th intercostal space and the scapular line into the target (middle) major calyx under ultrasonographic guidance (Siemens Healthcare, Munich, Germany). The puncture was deemed successful based on the outflow of urine upon the withdrawal of the core needle. A 0.035-inch zebra guidewire (uroVision $\mathrm{GmbH}$, Bad Aibling, Germany) was inserted through the needle sheath. Using a dagger knife, a small incision was made in the skin and subcutaneous fascia to retract the needle sheath. An introducer set (uroVision $\mathrm{GmbH}$, Bad Aibling, Germany) was used to dilate the renal access tract from $8 \mathrm{~F}$ to $12 \mathrm{~F}$. The dilator was withdrawn, and the Peel-away sheath (uroVision $\mathrm{GmbH}$, Bad Aibling, Germany) was maintained in the access route. A 9.8F dual-channel ureteroscope (Richard Wolf Medical Instruments Corporation, Vernon 
Hills, IL) was advanced into the renal pelvis and upper ureteral segment. A transpyelic ballistic lithotriptor (Electro Medical Systems, Nyon, Switzerland) was used to fragment the calculi in a stepwise fashion, and the stone fragments were completely removed by the combination of grasping and flushing. A double-J ureteral stent (uroVision $\mathrm{GmbH}$, Bad Aibling, Germany) was placed antegrade, and a percutaneous nephrostomy tube was placed.

Table 1 Clinical and surgical characteristics of scoliotic patients undergoing MPCNL.

\begin{tabular}{lcc}
\hline & Patient No. 1 & Patient No. 2 \\
\hline Age $($ year $)$ & 51 & 48 \\
Height $(\mathrm{cm})$ & 156 & 140 \\
Body mass $(\mathrm{kg})$ & 50 & 35 \\
Body mass index $\left(\mathrm{kg} / \mathrm{m}^{2}\right)$ & 20.5 & 17.9 \\
History of flank pain $(\mathrm{month})$ & 1 & 24 \\
Duration of aggravation & 3 days & 2 months \\
Calculus burden $\left(\mathrm{mm}^{2}\right)$ & 410 & 500 \\
Location of calculi & Right renal pelvis and upper & Left renal pelvis \\
Direction of spinal curvature & ureteral segment $(\mathrm{L3}$ level) & \\
Cobb angle $\left(^{\circ}\right)$ & Left & Left \\
Operative time (min) & 51 & 58 \\
Length of hospital stay (day) & 70 & 40 \\
Stone analysis & 10 & 9 \\
& Calcium oxalate mixed with & Calcium oxalate \\
\hline
\end{tabular}

\section{Postoperative Care and Follow-up}

Follow-up KUB radiography was performed on postoperative day (POD) 4, and the nephrostomy tube was removed on POD 5 if the double-J stent was appropriately positioned and no stone remnants were present. The urethral catheter was maintained until POD 7-8 to minimize the occurrence of ureteral reflux and ascending urosepsis caused by postoperative urinary retention, and the patients were discharged in the absence of fever or other discomfort. Patients were instructed to drink a set amount of water and modify their diets based on the stone analysis results. The double-J stent was removed using ureteroscopy seven weeks following the MPCNL. KUB, IVU, and non-contrast CT radiographs were ordered at three-month intervals.

\section{Results}

\section{Patient Characteristics}

The routine preoperative laboratory, including hematology, biochemistry, coagulation test, chest X-ray, electrocardiography, and pulmonary function test, showed no significant clinically abnormalities. The preoperative metabolic workup did not identify any clinically significant metabolic abnormalities.

A single tract was required in the ten patients. Percutaneous renal access was successfully established using ultrasonographic guidance. The entire surgery lasted approximately 40-60 min, and the time for establishing percutaneous access was 15 min in Patients, respectively. The urinary calculi were completely removed in one session of pneumatic lithotripsy along with stone grasping and flushing. The double-J stent was appropriately indwelled in ten patients. No urinary tract bleeding or perforation occurred in MPCNL. 
The ten patients had a successful recovery from MPCNL. The patients resumed oral intake on POD 2 and started off-bed activities on POD 3. There was no need for prolonged placement of the nephrostomy tube or the double-J stent. Thus, no patient required a second MPCNL or any other auxiliary intervention. The ten patients had stable renal function throughout the hospitalization and the follow-up period.

\section{Discussion}

ESWL is considered the least invasive modality for the treatment of urinary calculosis, but it has a higher incidence of lower stone recurrence compared to RUS or MPCNL[11]. ESWL is preferred for the lithotripsy of renal calculi between $4 \mathrm{~mm}$ and $20 \mathrm{~mm}$ in diameter, and is less successful for the fragmented ureteral stones[12]. MPCNL is a modified version of PCNL that has been used extensively in China over the past two decades[15]. The popularity of MPCNL lies in its reported favorable procedural safety, however it has limited indications due to the small percutaneous tract (11-20F versus 26-34F)[13]. As such, MPCNL is primarily used in pediatric patients or adult patients with small stones or as a secondary technique used to supplement conventional PCNL[14]. Advancements in MPCNL equipment and techniques have helped broaden the indication of MPCNL to any UTUL requiring PCNL intervention, as suggested by Guangzhou and colleagues[15]. Using multiple tracts, MPCNL allows for the removal of staghorn calculi or complex renal calculi[16].

The appropriate choice of the interventional modality depends primarily on the stone burden, stone location, and the presence of concomitant anomalies, such as scoliosis. [9]Scoliosis is a rare medical condition that is characterized by the abnormal lateral curvature of the spinal column. It is typically classified as congenital, idiopathic and neuromuscular. Idiopathic scoliosis accounts for the majority of cases (70\%), and the prevalence is higher in females (M/F, 1:4)[17]. Congenital scoliosis is frequently associated with syndromes and anomalies of other systems, including neurological, cardiovascular, and genitourinary systems. Severe scoliosis may compromise patients' cardiopulmonary function and consequently increase the surgical risks of non-orthopedic procedures[18]. Urinary morbidity can occur secondary to scoliosis, including urinary deviation, urosepsis, calculosis, and renal impairment. The prophylactic use of wide-spectrum antibiotics is recommended even in the case of culture-negative urosepsis. ESWL is less useful in scoliotic patients as it is technically challenging to localize urinary calculi.

The renal and/or upper ureteral calculi were fragmented by the application of pneumatic pressure, and removed by pulsating and pressurized irrigation rather than by grasping with delicate forceps used in MPCNL. However, this forceful pulse flow raises a safety concern that the high intrapelvic pressure in MPCNL may cause backflow into the collecting system via vascular, lymphatic, and mesenchymal routes, and further subject patients to a higher risk of urosepsis[16]. In contrast to this concern, some suggest that MPCNL is safe, as the renal pelvic pressure remains lower than 30 $\mathrm{mmHg}$, a pressure that usually leads to backflow. [17].Multiple tracts can be used to minimize backflow, thus reducing intrapelvic pressure[18]. The multi-tract technique shortens the procedure duration, improves stone clearance, and facilitates the flushing out of fragmented stones by irrigation. [19] Although the use of MPCNL has broadened, it not suitable for the removal of stones greater than $30 \mathrm{~mm}$ in diameter, as it has a high-risk of leaving residual particles.

The presence of musculoskeletal deformities inevitably complicates the endourological procedure[9]. Like any other MIS technique, MPCNL requires the 
operators to have a good knowledge of local anatomy. The appropriate puncture into the collecting system is the most crucial for a successful MPCNL and greatest risk occurs during the percutaneous manipulation[15]. It is technically challenging to establish the nephrolithotomy access in scoliotic patients, especially in those with a larger lateral spinal curvature. The stones located in the upper major calyx are relatively less accessible than those located in the pelvis or the ureter. The percutaneous puncture into the stone-bearing major calyx or pelvis located ipsilateral to the spinal curvature is relatively easier as compared to that contralateral as this access is less likely to involve intestines or other intraperitoneal organs. A thorough preoperative radiological assessment can characterize the anatomy of the diseased kidney in relation to the surrounding organs, and help minimize the occurrence of iatrogenic injuries. An abdominal contrast-enhanced CT scan is recommended to minimize the risk of colonic penetration. Using ultrasonographic guidance, the calyx puncture was completed with a single attempt in the two patients. In contrast, Kara et al. [9] positioned the puncture site using retrograde contrast fluoroscopy, and although this procedure is less convenient and more invasive, they reported no adverse event. However, the technical difficulty depends on the severity of complicating hydronephrosis rather than the location of stones.

Despite MPCNL's minimally invasive advantages, it is subject to some complications[15] .The primary complications of MPCNL consist of visceral injury, renal vascular injury, urinary tract perforation/leakage, urosepsis, and hydrothorax caused by the percutaneous puncture at the 11th intercostal space. As reported by Kara et al., [9] the most common and serious complication following PCNL is renal hemorrhage, which requires blood transfusions in severe cases. The operator's experience is thought to be the most significant predictor of urinary tract bleeding[9].

Our results show that MPCNL is an effective and safe minimally invasive modality for the treatment of urolithiasis in patients. Ultrasonographic guidance will not only improve MPCNL performance, but also reduce the procedural risks. Other MIS approaches, such as ESWL and ureteroscopy, might also be attempted or used as adjuvants to MPCNL.

\section{References}

[1]Wignall GR, Canales BK, Denstedt JD, et al. Minimally invasive approaches to upper urinary tract urolithiasis. Urol Clin North Am 2008;35:441-54

[2]Tiselius HG, Ackermann D, Alken P, et al. Guidelines on urolithiasis. Eur Urol 2001;40:362-71.

[3]Patrick H, Gunnar WN, Patrick K, et al. Does open stone surgery still play a role in the treatment of urolithiasis? Data of a primary urolithiasis center. J Endourol 2009;23:1209-12.

[4]Nguyen HD, Tan YH, Wong MY. Percutaneous nephrolithotomy in the management of complex upper urinary tract calculi: the Singapore General Hospital experience. Ann Acad Med Singapore 2002;31:516-9.

[5]Wyatt J, Kolettis PN, Burns JR. Treatment outcomes for percutaneous nephrolithotomy in renal allografts. J Endourol 2009;23:1821-4.

[6]Gupta NP, Mishra S, Seth A, et al. Percutaneous nephrolithotomy in abnormal kidneys: single-center experience. Urology 2009;73:710-4; discussion 714-5. 
[7]el-Nahas AR, Eraky I, el-Assmy AM, et al. Percutaneous treatment of large upper tract stones after urinary diversion. Urology 2006;68:500-4.

[8]Mohanty S, Kumar N. Patterns of presentation of congenital scoliosis. J Orthop Surg (Hong Kong) 2000;8:33-7.

[9]Kara C, Resorlu B, Ozyuvali E, et al. Is percutaneous nephrolithotomy suitable for patients with Scoliosis: Single-center experience. Urology 2011;78:37-42.

[10]Wu K, Li X, Yuan J, et al. Mini nephrostomy with ureteroscopic lithotripsy for staghorn stones. Acad J Guangzhou Med Coll 1993;2:13-4.

[11]Srisubat A, Potisat S, Lojanapiwat B, et al. Extracorporeal shock wave lithotripsy (ESWL) versus percutaneous nephrolithotomy (PCNL) or retrograde intrarenal surgery (RIRS) for kidney stones. Cochrane Database Syst Rev 2009;(4):CD007044.

[12]Abe T, Akakura K, Kawaguchi M, et al. Outcomes of shockwave lithotripsy for upper urinary-tract stones: a large-scale study at a single institution. J Endourol 2005; 19:768-73.

[13]Cheng F, Yu W, Zhang X, et al. Minimally invasive tract in percutaneous nephrolithotomy for renal stones. J Endourol 2010;24:1579-82.

[14]Jackman SV, Docimo SG, Cadeddu JA, et al. The "mini-perc'" technique: A less invasive alternative to percutaneous nephrolithotomy. World J Urol 1998;16:371-4.

[15] Li X, He Z, Wu K, et al. Chinese minimally invasive percutaneous nephrolithotomy: the Guangzhou experience. J Endourol 2009;23:1693-7.

[16]Zhong W, Zeng G, Wu W, et al. Minimally invasive percutaneous nephrolithotomy with multiple mini tracts in a single session in treating staghorn calculi. Urol Res 2011;39:117-22.

[17]Weinstein SL, Dolan LA, Spratt KF, et al. Health and function of patients with untreated idiopathic scoliosis: a 50-year natural history study. JAMA 2003;289:559-67.

[18]Giampietro PF, Blank RD, Raggio CL, et al. Congenital and idiopathic scoliosis: clinical and genetic aspects. Clin Med Res 2003;1:125-36.

[19]Tomaszewski JJ, Ortiz TD, Gayed BA, et al. Renal access by urologist or radiologist during percutaneous nephrolithotomy. J Endourol 2010;24:1733-7. 